\title{
OPTICAL INVESTIGATION TO MINIMIZE THE ELECTRON BUNCH ARRIVAL-TIME JITTER BETWEEN FEMTOSECOND LASER PULSES AND ELECTRON BUNCHES FOR LASER-DRIVEN PLASMA WAKEFIELD ACCELERATORS*
}

\author{
S. Mattiello ${ }^{\dagger}$, Andreas Penirschke, Technische Hochschule Mittelhessen, Friedberg, Germany \\ Holger Schlarb, DESY, Hamburg, Germany
}

\section{Abstract}

In a laser driven plasma based particle accelerators a stable synchronization of the electron bunch and of the plasma wakefield in the range of less than $2 \mathrm{fs}$ is necessary in order to optimize the acceleration. For this purpose we are developing a new shot to shot feedback system with a time resolution of less than $1 \mathrm{fs}$. As a first step, stable $\mathrm{THz}$ pulses are generated by optical rectification of a fraction of the plasma generating high energy laser pulses in a nonlinear lithium niobate crystal. It is planed that the generated $\mathrm{THz}$ pulses will energy modulate the electron bunches shot to shot before the plasma to achieve the time resolution of $1 \mathrm{fs}$. In this contribution we systematically investigate the influence of the optical properties as well as the theoretical description of the $\mathrm{THz}$ generation on the conversion efficiency of the generation of short $\mathrm{THz}$ pulses, in undepleted approximation. We compare different approximations for the modeling of the generation dynamics and of the dielectric function in order to investigate the importance of a detailed description of the optical properties. First results by considering intensity decreasing of the laser pump will be presented.

\section{INTRODUCTION}

Particle accelerators are important tools for fundamental research as well for the industry and human life. In fact, they allow to achieve crucial new discoveries, e.g. the Higgs boson, to test and develop the standard model and to deeply understand the strong interaction and in particular the properties of the quark gluon plasma. On the other side, accelerators have several applications in material science, biology, medicine, industry and for security purposes and point-to point communications. As examples we mention the systematic investigation of basic materials using ions beams in order to achieve new technologies, the repair of complex DNA lesions as well as cancer treatment, where the high-energy particle beams can be targeted to destroy tumors [1,2].

The technology of standard accelerators is coming to its limit given by the physical-chemical properties of the material used for the construction as well as by the huge size of new accelerators and by the financial costs.

Because of their extremely large accelerating electric fields, plasma-based particle accelerators driven by either

\footnotetext{
* The work of S. Mattiello is supported by the German Federal Ministry of Education and Research (BMBF) under contract no. 05K16ROA.

$\dagger$ stefano.mattiello@iem.thm.de
}

lasers or particle beams allow to overcome these problems. In this method, known as plasma wakefield acceleration (PWA), the accelerating gradients in plasmas can be 3-4 orders of magnitude higher than in conventional accelerators [3].

The period of these fields is in the range of $10 \mathrm{fs}$ to $100 \mathrm{fs}$ so that for an optimization of the acceleration a stable synchronization of the electron bunch and of the plasma wakefield in the range of few femtoseconds is necessary. Consequently, the minimizing the electron bunch arrival-time jitter becomes a central point for the realization of these accelerators. We are planning a new shot to shot feedback system which should be able to synchronize the electron bunch with the plasma exciting laser pulse with a time resolution of less than $1 \mathrm{fs}$.

In a first step, stable Terahertz (THz) pulses should be performed by optical rectification (OR) of high energy laser pulses in a nonlinear crystal. These pulses allow an energy modulation in the modulator placed in a chicane of the electron bunch in order to achieve the required resolution. This paper focuses on the first step of the feedback system, i.e. the generation of $\mathrm{THz}$ pulses.

For our purpose an efficient and stable generation of $\mathrm{THz}$ pulses is a challenging task. Consequently, the main focus is to maximize the conversion efficiency of the $\mathrm{THz}$ generation defined in term of the $\mathrm{THz}$ frequency component of the electric field as [4],

$$
\eta=\frac{\pi \epsilon_{0} c \int_{0}^{\infty} \mathrm{d} \Omega n(\Omega)|E(\Omega, z)|^{2}}{F_{\mathrm{p}}},
$$

where $F_{\mathrm{p}}$ and $\varepsilon_{0}$ indicate the pump fluence and the vacuum dielectric constant respectively.

For optical rectification the selection of the nonlinear material is fundamental aspect. Because of its high nonlinear optic coefficient, lithium niobate $\left(\mathrm{LiNbO}_{3}, \mathrm{LN}\right)$ is a suitable material for $\mathrm{THz}$ generation.

The optical properties of the material and in particular the details of the adsorption of the $\mathrm{THz}$ radiation and material dispersion effects are important for the optimization of the efficiency. In this contribution we investigate the influence of the optical properties, where we focus on a periodically poled lithium niobate crystal (PPLN) [4].

The paper is organized as follows. First, we derive the general equations for the description of the $\mathrm{THz}$ generation and then we present the calculation of the optical properties of the material and the different approximations for the solution of the $\mathrm{THz}$ generation. Therefore we investigate in 
this framework the role of the optical properties of PPLN on the conversion efficiency of the generation of $\mathrm{THz}$ pulses. A treatment of the influence on $\eta$ of the intensity decreasing of the laser pump is presented. The conclusions finalize this work.

\section{DERIVATION OF THE THZ GENERATION EQUATIONS}

Starting from the Maxwell equations the one dimensional wave equation for the optical and $\mathrm{THz}$ waves can be derived as $[5,6]$,

$$
\left(\frac{\partial^{2}}{\partial z^{2}}+\epsilon \mu \frac{\partial^{2}}{\partial t^{2}}-\mu \sigma \frac{\partial}{\partial t}\right) E_{\mathrm{tot}}(t, z)=-\mu \frac{\partial P_{\mathrm{NL}}(t, z)}{\partial t^{2}}
$$

$\epsilon$ denotes is the dielectric constant, $\mu$ is the magnetic permeability of vacuum, $\sigma$ denotes the electrical conductivity and $P_{\mathrm{NL}}$ is the nonlinear polarization.

The electric field $E_{\text {tot }}(t, z)$ is the sum of the electric field of an optical frequency component (laser pulse), $E_{\mathrm{p}}(t, z)$ and a $\mathrm{THz}$ frequency component, $E(t, z)$. Consequently the equation of motion can be treated as a set of coupled wave equation for $E_{\mathrm{p}}$ and $E$.

Several works consider only the equation for the $\mathrm{THz}$ component, i.e. the depletion of the laser pump is neglected [7-9]. In this undepleted approximation the generation of the $\mathrm{THz}$ wave can be expressed in frequency domain as

$$
\left(\frac{\partial^{2}}{\partial z^{2}}+\frac{\Omega^{2}}{c^{2}} \varepsilon(\Omega)\right) E(z, \Omega)=-\mu_{0} \omega^{2} P_{\mathrm{NL}}(\Omega, z)
$$

where $\varepsilon(\Omega)$ is the generalized (complex) dielectric function, in which the contribution of the electric conductivity is encoded. The polarization reads

$$
P_{\mathrm{NL}}(\Omega, z)=\varepsilon_{0} \chi^{(2)} \int_{0}^{\infty} \mathrm{d} \omega A_{\mathrm{p}}(\omega+\Omega, z) A_{\mathrm{p}}^{*}(\omega, z) \mathrm{e}^{l(k(\Omega+\omega)-k(\omega)) z}
$$

where the parameter $\chi_{\text {eff }}^{(2)}(z)$ is the effective second order nonlinear susceptibility which can be a function of the spatial coordinate $z$. Here we used that for arbitrary $z$ holds

$$
E_{\mathrm{p}}(\omega, z)=A_{\mathrm{p}}(\omega) \mathrm{e}^{-\imath k(\omega) z}
$$

where $k(\omega)$ denotes the module of the wave vector.

These equations show that the optical properties of the material in two different frequency ranges are the relevant physical inputs for the $\mathrm{THz}$ generation, i.e. for the dielectric function $\varepsilon(\Omega)$ in the THz region and for the module of the wave vector $k(\omega)$, which is connected to the dielectric function at the frequency $\omega$.

Consequently, before we discuss the solutions of Eq. (3), we present the description of the optical properties for the two different frequency ranges.

\section{MODELING THE MATERIAL PROPERTIES}

In order to determine the optical properties, we have to calculate the complex dielectric function $\varepsilon(\omega)$ of the material. In general the relation between $\varepsilon(\omega)$ and the wave vector, the refractive index and the adsorption coefficient is given by

$$
n(\omega)=\frac{k(\Omega) c}{\Omega}=\mathfrak{R} \sqrt{\varepsilon(\omega)} \quad \text { and } \quad \alpha(\omega)=\frac{2 \Omega}{c} \mathfrak{J} \sqrt{\varepsilon(\omega)} .
$$

Because in the polarization integral the optical properties enter in term of the expression $k(\Omega+\omega)-k(\omega)$, only the real part of the dielectric function is relevant in the frequency region around $\omega_{0}$.

In literature, several works [7-9] perform a linear approx imation of this term, i.e.

$$
k(\Omega+\omega)-k(\omega) \approx \frac{n_{\mathrm{gr}}}{c} \Omega,
$$

which is independent on the integration variable, so that for a Gaussian enveloped laser pulse $P_{\mathrm{NL}}$ can be expressed analytically.

Nevertheless, the dispersion plays an important role that cannot be neglected in our contribution. In order to systematically implement this effect, we describe the refractive index squared as function of the frequency using the Sellmeier equation given in Ref. [10]. For the $\mathrm{THz}$ frequency region we use a physical motivated description for the dielectric function based on an oscillator model, i.e.

$$
\varepsilon(\Omega)=\varepsilon_{\infty}+\sum_{j} \frac{S_{j} \Omega_{j}^{2}}{\Omega_{j}^{2}-\Omega^{2}-i \Omega \Gamma_{j}},
$$

where the summation over the lattice oscillators with the strength $S_{j}$, angular resonance frequency $\Omega_{j}$ and resonance width $\Gamma_{j}$ is performed and the bound electron contribution to the dielectric function is denoted as $\varepsilon_{\infty}$ [11]. In this contribution we use the parameter set Schall(1999) from Ref. [11] as well as our parameter Mattiello(2017) [2], in order to investigate the influence of the dielectric function on $\eta$.

Additionally, free carries (FC) are generated by the pump adsorption in the material $[8,9]$. Then, the dielectric function has to be modified, so that it includes systematically the FC contribution. We use a dielectric function, in which the contribution of the free carries is implemented along the line of a Drude model

$$
\varepsilon_{\mathrm{tot}}(\Omega)=\varepsilon_{\mathrm{osc}}(\Omega)-\frac{\omega_{\mathrm{pl}}^{2}}{\Omega^{2}+i \Omega / \tau_{\mathrm{sc}}} .
$$

Hereby $\tau_{\mathrm{sc}}=200 \mathrm{fs}$ [9] is the electron scattering time and the plasma frequency $\omega_{\mathrm{pl}}^{2}$ is related to the density of free charge carries $\rho_{\mathrm{FC}}$ by

$$
\rho_{\mathrm{FC}=} \frac{\varepsilon_{0} \varepsilon_{\infty}^{\mathrm{FC}} m_{\mathrm{eff}}}{e^{2}} \omega_{\mathrm{pl}}^{2},
$$


where $e$ and $m_{\mathrm{eff}}=0.25 m_{\mathrm{e}}$ are the electron charge and effective masse respectively [9]. Herby $m_{\mathrm{e}}$ indicates the electron mass. Instead to systematically modify the dielectric function, in several works a simple sum approximation is used,

$$
\alpha(\Omega)=\alpha_{\varepsilon}(\Omega)+\alpha_{\mathrm{FC}}(\Omega)
$$

where the two terms are calculated separately starting from a dielectric function without free carries and the dielectric function of a Drude model respectively $[8,9]$.

For PPLN, the physical relevant input $\rho_{\mathrm{FC}}$ can be described by the three-photon-adsorption process(3PA) of the pump beam in the medium [9]

$$
\varrho_{3 \mathrm{PA}}\left(I_{\mathrm{p}}\right)=\frac{\tau \lambda_{0}}{h c} \frac{1}{3} \gamma_{3} I_{\mathrm{p}}^{3}
$$

with $\gamma_{3}=1.3 \times 10^{-4} \mathrm{~cm}^{3} \mathrm{GW}^{-2}$. Here $I_{\mathrm{p}}$ is the timeaveraged pump pulse intensity over pulse duration $\tau, \lambda_{0}$ is the pump central wavelength and $\gamma_{3}$ denote the absorption coefficients for three-photon process. However, by further increasing pump fluence, saturation of absorption contribution of FC seems to occur, leading to an increase of $\mathrm{THz}$ conversion efficiency. This behavior was observed by LN crystals [9]. To investigate this effect we parametrize the saturation with the following phenomenological expression

$$
\varrho\left(I_{\mathrm{p}}\right)=\varrho_{\mathrm{s}}-A \mathrm{e}^{-a\left(I_{\mathrm{p}}-I_{0}\right)},
$$

setting the saturation concentration of free carriers in LN crystal to $\varrho_{\mathrm{s}} \approx 4 \times 10^{14} \mathrm{~cm}^{-3}$ at pump fluence $F_{\mathrm{s}}=$ $50 \mathrm{~mJ} \mathrm{~cm}^{-2}$ [9]. Hereby $I_{0}$ denotes the intensity of the laser, where this saturation formula set on.

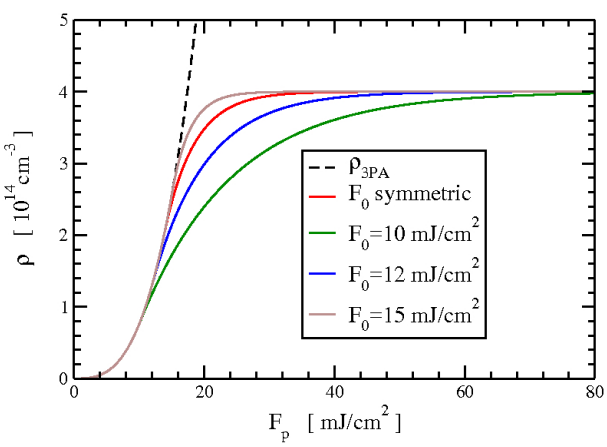

Figure 1: The density of free charge carries as function of the fluence in strict 3PA (dashed black line) and for different values of $F_{0}$ for a Gaussian pulse with duration $\tau_{\mathrm{FWHM}}=$ $25 \mathrm{fs}$ and $\lambda 0=1030 \mathrm{~nm}$ as indicated.

The Fig. 1 shows the density of free charge carriers as function of the fluence in strict 3PA (dashed line) and for different values of $F_{0}$ for a Gaussian pulse with duration $\tau_{\mathrm{FWHM}}=$ $25 \mathrm{fs}$ and $\lambda 0=1030 \mathrm{~nm}$ as indicated. Herby we used that in the case of Gaussian pulses, fluence and intensity are proportional. With symmetric we indicate the parametrization, in which the transition occurs at the half of the saturation density, i.e. $\varrho_{3 \mathrm{PA}}\left(F_{0}\right)=\varrho_{\mathrm{s}} / 2=2 \times 10^{14} \mathrm{~cm}^{-3}$. We expect that the different parametrizations for the saturation lead to sizeable different behaviors of the efficiency as function of the fluence. We discuss this point in the section RESULTS.

\section{MODELING THE THZ GENERATION}

Not only the different implementation of the optical properties and the approximations (full Sellmeier equation or linear approximation for the dispersion, systematic implementation of the free charge carries, different parametrization of the saturation behavior), but also the way to solve the equation of motion (3) lead to diverse results for the conversion efficiency.

\section{Slope Varying Approximation}

Almost all investigations confine themselves to a slope varying approximation (SVA) [4,7-9], in which neglecting the second spatial derivate of the amplitude leads to a linear differential equation of the first order,

$$
\frac{\partial}{\partial z} A(\Omega, z)=-\frac{\alpha(\Omega)}{2} A(\Omega, z)-\imath \frac{\Omega}{2 k(\Omega) c^{2}} \frac{P_{\mathrm{NL}}(\Omega, z)}{\varepsilon_{0}} \mathrm{e}^{\imath k(\Omega) z} .
$$

In the linear approximation for the polarization integral, i.e. if Eq. (7) is used, the absolute value of the field squared can be expressed by

$$
\begin{aligned}
|A(\Omega, z)|^{2} & =\frac{\Omega^{2} \tilde{P}_{\mathrm{NL}}^{2}(\Omega, z)}{4 c^{2} n^{2}(\Omega) \varepsilon_{0}^{2}} \frac{1}{\left(\Delta k_{0}\right)^{2}+a_{\mathrm{T}}^{2}(\Omega) / 4} \\
& \times\left(\left(1-\mathrm{e}^{-\frac{\alpha(\Omega) z}{2}}\right)^{2}+4 \mathrm{e}^{-\frac{\alpha(\Omega) z}{2}} \sin ^{2}\left(\frac{\Delta k_{0} z}{2}\right)\right),
\end{aligned}
$$

where the effective nonlinear polarization $\tilde{P}_{\mathrm{NL}}$ is given by

$$
\tilde{P}_{\mathrm{NL}}(\Omega, z)=\varepsilon_{0} \chi_{\text {eff }}^{(2)}(z) \int_{0}^{\infty} \mathrm{d} \omega A_{\mathrm{p}}(\omega+\Omega, z) A_{\mathrm{p}}^{*}(\omega, z) .
$$

Here the mismatching $\Delta k$, which for PPLN is in general defined by

$$
\Delta k=k(\Omega+\omega)-k(\omega)-k(\Omega)+\frac{2 \pi}{\Lambda},
$$

becomes, using Eq. (7), $\Delta k \longrightarrow \Delta k_{0}=\frac{\Omega}{c}\left(n_{\mathrm{opt}}^{\mathrm{gr}}-n(\Omega)\right)+$ $\frac{2 \pi}{\Lambda}$, where $\Lambda=237.74 \mu \mathrm{m}$ is the quasi-phase-matching orientation-reversal period [4].

In general, beyond the linear approximation in the polarization integral, the amplitude can be written as

$$
\begin{aligned}
A(\Omega, z) & =-l \frac{\Omega^{2} \chi_{\mathrm{eff}}^{(2)}}{2 k(\Omega) c} \int_{0}^{\infty} \mathrm{d} \omega\left[\frac{A_{\mathrm{p}}(\omega+\Omega) A_{\mathrm{p}}^{*}(\omega)}{\alpha / 2-\imath \Delta k}\right. \\
& \left.\times\left(\mathrm{e}^{-\imath \Delta k z}-\mathrm{e}^{\alpha z / 2}\right)\right]
\end{aligned}
$$

where $\Delta k$ is a function of the integration variable $\omega$. If the quantity

$$
\tilde{J}_{1}(\omega, \Omega)=\frac{A_{\mathrm{p}}(\omega+\Omega) A_{\mathrm{p}}^{*}(\omega)}{\alpha^{2} / 4+(\Delta k)^{2}}
$$

is real, $A$ can be expressed as linear combinations of integrals involving $\tilde{J}_{1}(\omega, \Omega)[2]$. Consequently, a(semi)analytical calculation of the efficiency $\eta$ without any approximation for the dispersion relation in the optical range can be performed. 


\section{Solution for the Second Order Equation}

Assuming a forward propagation of the $\mathrm{THz}$ wave a formal solution for Eq. (3) can be given as

$$
\begin{aligned}
E(\Omega, z) & =\frac{\Omega^{2} \chi_{\mathrm{eff}}^{(2)}}{c^{2}} \mathrm{e}^{-i k(\Omega) z} \int_{0}^{\infty} \mathrm{d} \omega\left[\left(\mathrm{e}^{-\imath \Delta k z}-\mathrm{e}^{\alpha z / 2}\right)\right. \\
& \left.\times \frac{A_{\mathrm{p}}(\omega+\Omega) A_{\mathrm{p}}^{*}(\omega)}{\left(\Delta k_{-}+\imath \alpha / 2\right)\left(\Delta k_{+}-\imath \alpha / 2\right)}\right]
\end{aligned}
$$

where the generalized mismatch vectors are given for PPLN by

$$
\Delta k_{ \pm}=k(\Omega+\omega)-k(\omega)+\frac{2 \pi}{\Lambda} \pm k(\Omega)
$$

As in the case of the SVA, using the linear approximation for the polarization function an analytic expression for the absolute value of the field squared can be given by

$$
\begin{aligned}
|A(\Omega, z)|^{2} & =\frac{\Omega^{4} \tilde{P}_{\mathrm{NL}}^{2}(\Omega, z)}{c^{4}} \frac{1}{\left(\Delta k_{-}\right)^{2}+\alpha^{2} / 4} \frac{g_{1}^{2}+g_{2}^{2}}{\left(\Delta k_{+}\right)^{2}+\alpha^{2} / 4} \\
\times & \left(\left(1-\mathrm{e}^{-\frac{\alpha(\Omega) z}{2}}\right)^{2}+4 \mathrm{e}^{-\frac{\alpha(\Omega) z}{2}} \sin ^{2}\left(\frac{\Delta k_{0} z}{2}\right)\right),(22)
\end{aligned}
$$

where we define $g_{1}=(k(\Omega+\omega)-k(\omega))^{2}-k^{2}(\Omega)+\alpha^{2} / 4$ and $g_{2}=-\alpha k(\Omega)$.

Similarly, if for the dispersion relation the Sellmeier equation is used, under the requirement that

$$
\tilde{J}_{2}(\omega, \Omega)=\frac{A_{\mathrm{p}}(\omega+\Omega) A_{\mathrm{p}}^{*}(\omega)}{\alpha^{2} / 4+\left(\Delta k_{-}\right)^{2}} \frac{1}{\alpha^{2} / 4+\left(\Delta k_{+}\right)^{2}} \in \mathfrak{R},
$$

we calculate $\eta$ semianalitically, because $E$ can be expressed as linear combination of integrals involving $\tilde{J}_{2}(\omega, \Omega)$ [2].

\section{RESULTS}

In the following we consider a periodic polarized congruent lithium niobate crystal with $\chi_{\text {eff }}^{(2)}=336 \mathrm{pm} \mathrm{V}^{-1}$ at room temperature with a Gaussian laser beam pulse with central wave length $\lambda 0=1030 \mathrm{~nm}$ and a pulse duration at full width of half-maximum $\tau_{\mathrm{FWHM}}=25 \mathrm{fs}[4,7,9]$. In this case $\tilde{J}_{1}$ as well as $\tilde{J}_{2}$ are real and the reduced nonlinear polarization reads

$$
\tilde{P}_{\mathrm{NL}}(\Omega, z) \equiv \tilde{P}_{\mathrm{NL}}(\Omega)=\varepsilon_{0} \chi_{\mathrm{eff}}^{(2)} \frac{E_{0}^{2} \tau}{2 \sqrt{2 \pi}} \exp \left(-\frac{-\tau^{2} \Omega^{2}}{8}\right)
$$

where $E_{0}$ is the peak value of the electric field, $\tau$ is given by $\tau=(2 \sqrt{2})^{-1 / 2} \tau_{\text {FWHM }}$ and $n_{0}=n\left(\lambda_{0}\right)$ is calculated using the Sellmeier equation.

We firstly present our results obtained in SVA. In Fig. 2 we show the conversion efficiency $\eta$ for a fixed pump fluence $F_{\mathrm{p}}=5 \mathrm{~mJ} \mathrm{~cm}^{-2}$ as function of the crystal length $L$ using different approximation for the optical properties and the parameters set Schall(2009).

The solid and dashed lines refer to the calculation using the Sellmeier equation for the optical dispersion and the linear approximation in the polarization integral, see Eq. (7), respectively. The colors indicate the different modeling of the

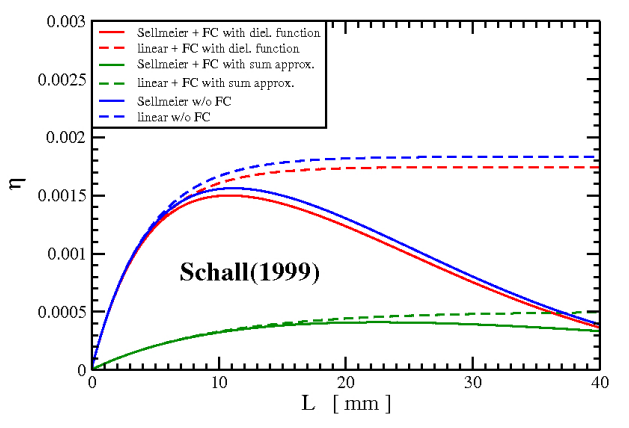

Figure 2: The conversion efficiency $\eta$ for a fixed pump fluence $F_{\mathrm{p}}=5 \mathrm{~mJ} \mathrm{~cm}^{-2}$ as function of the crystal length $L$ using different approximation for the optical properties and the parameters of Schall [11] within SVA.

dielectric function: red indicates the systematic implementation of the free charge carries contribution using the dielectric function of Eq. (9), green denotes the approximation of the FC contribution as sum of the adsorption coefficient of the oscillator and of the Drude model, see Eq. (11), and the blue lines indicate the results by neglecting the free charge carries, i.e. using for the dielectric function of Eq. (8).

The results with the linear approximation for the dispersion relation in the optical range show a saturation of $\eta$ for large crystal lengths. The functional behavior of the efficiency changes by considering their full optical dispersion given by Sellmeier equation.

For $L>10 \mathrm{~mm} \eta$ is a decreasing function of the crystal length. For the calculation with the systematically modified dielectric function we note non monotonic behavior of $\eta(z)$, that is in line with the depleted results of Ref. [4]. This agreement can be understood, if we consider that the consistent implementation of the free charge carries can be viewed as a coupling of the laser pulse with the optical properties via the plasma frequency, which directly depends on the FC density.

In general, we find sizeable modifications, if the contribution of the free carries is implemented using the sum approximation. This strong suppression of the efficiency in this approximation can be an indication that such a modeling of the FC could lead to unphysical underestimation of the $\eta$. Additionally, the calculation of $\eta$ for a fixed pump fluence $F_{\mathrm{p}}=5 \mathrm{~mJ} \mathrm{~cm}{ }^{-2}$ as function of the crystal length $L$ using the parameters set Mattiello(2017) show a quantitative approximate independence of the results for the efficiency from the oscillator models for small pump fluence.

For the investigation of the dependence of $\eta$ from the parametrization of the density of the free charge carries as well as from the choice of the dielectric function at higher pump fluence we consider the efficiency as function of the pulse fluence at fixed crystal length using both parameters sets. In Fig. 3 we show $\eta$ for a crystal length of $L=5 \mathrm{~mm}$ as function of $F_{\mathrm{p}}$ using the Sellmeier equation for the polarization integral and different parametrization of the FC density, labeled by $F_{0}$ as indicated. For comparison, we plot as dashed line the results for the efficiency calculated with- 


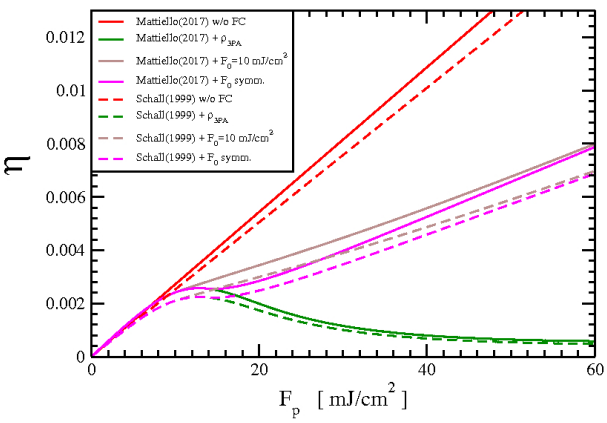

Figure 3: Comparison of the conversion efficiency $\eta$ for a crystal length of $5 \mathrm{~mm}$ as function of $F_{\mathrm{p}}$ calculated with our parameters [2](solid line) and with these of Schall [11] (dashed line) in SVA using the Sellmeier equation. The different parametrizations of the FC density are labeled by $F_{0}$ as indicated. As reference the efficiency by neglecting FC has been shown as solid and dashed red line respectively.

out FC contribution. The efficiencies calculated with our parameters [2] and these with the parameter of Schall [11] are shown as solid and dashed line respectively. If the FC contribution is neglected, the behavior of the efficiency as function of the fluence is linear. For unsaturated density of $\mathrm{FC}$, the $\mathrm{THz}$ conversion efficiency increases for small values of $F_{\mathrm{p}}$, show a local maximum and then decreases by increasing pump fluence.

A different behavior occurs for saturated $\varrho_{\mathrm{FC}}$. For $F_{0}>$ $10 \mathrm{~mJ} \mathrm{~cm}^{-2}$ we observe a local maximum an a local minimum of the efficiency, so that three different monotonic regimes, i.e. increasing, decreasing and again increasing behavior as function of the fluence, can be founded. For smaller values of $F_{0}$ he efficiency increases with increasing pump fluence. Nevertheless, the point, which for larger $F_{0}$ corresponds to the local maximum of $\eta$, becomes the value, where the slope of the increasing behavior changes. These findings show the importance of a dynamical description of the generation and saturation of the free charge carriers in order to explain in such an effective calculation the behavior of the THz generation efficiency. Additionally, we note sizeable differences between the results achieved using the two models for the oscillator part of the dielectric function.

This indicates the existence regions of value of the physical parameters, i.e. $L$ and $F_{\mathrm{p}}$, where $\eta$ strongly on the models of $\varepsilon_{\mathrm{osc}}$. Therefore, in particular for this range of crystal length and fluence, a better description of the optical properties is needed.

In order to investigate the deviation, which emerges by solving the equation of motion as differential equation of the second order, we consider the efficiency as function of the crystal length at fixed fluence. In Fig. 4 we compare the results for the conversion efficiency $\eta$ for a fixed pump fluence $F_{\mathrm{p}}=5 \mathrm{~mJ} \mathrm{~cm}^{-2}$ as function of the crystal length $L$ between the second order calculation (solid lines) and the SVA (dashed lines) using the parameters set Schall(1999) and our systematic implementation of FC using the dielectric function of Eq. (9). Using the linear approximation within

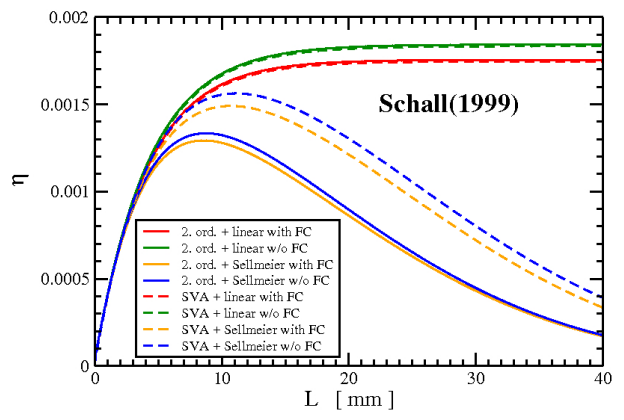

Figure 4: The conversion efficiency $\eta$ for a fixed pump fluence $F_{\mathrm{p}}=5 \mathrm{~mJ} \mathrm{~cm}^{-2}$ as function of the crystal length $L$ for the second order calculation (solid lines) and the SVA (dashed lines) using the parameters of Schall [11] labeled by different colors as indicated.

the polarization integral, only small deviations emerge. If the Sellmeier equation for the dispersion relation has been used, the quantitative deviations are sizeable. Therefore, at small fluences, the importance of the second order dynamic seems to be stronger than the effects of the free charge carries. On the other side, further investigations show that for large fluence, sizeable but small deviation emerge between the second order and SVA calculations. Consequently, we can argue, that, at high fluences, the value of $\eta$ is dominated by the details of the formation and saturation of FC and that the deviations coming from second order effects are small ${ }^{1}$.

\section{DECREASING OF THE PUMP INTENSITY}

We present now our first calculations of the influence on $\eta$ of the intensity decreasing of the laser pump induced by the three photon adsorption in SVA approximation with the linear approximation for the dispersion relation in the optical range. By neglecting the linear adsorption the intensity is

$$
I_{\mathrm{p}}(t, z)=I_{0}\left(t-t_{0}\right)\left(1+2 \gamma_{3} I_{0}^{2}\left(t-t_{0}\right)\right)^{-1 / 2}
$$

where $I_{0}(t)$ denotes the intensity at $z=0$ and the time shift is given by $t_{0}=n_{\mathrm{opt}}^{\mathrm{gr}} z / c$. After the Fourier Transformation of the Taylor expansion of the intensity we can write

$$
\begin{aligned}
I_{\mathrm{p}}(\Omega, z) & =\mathrm{e}^{-l q z} \sum_{n=0}^{\infty} \tilde{I}_{n} z^{n} \\
\tilde{I}_{n} & =\frac{u_{n}}{2 \sigma_{n} \sqrt{\pi}} \mathrm{e}^{-\frac{\Omega^{2}}{4 \sigma_{n}^{2}}}
\end{aligned}
$$

where we define $u_{n}=\left(\begin{array}{c}-1 / 2 \\ n\end{array}\right) I_{0}\left(2 \gamma_{3} I_{0}^{2}\right)^{n}, \sigma_{n}^{2}=\frac{2}{\tau^{2}}(2 n+1)$ and $q=\Omega n_{\mathrm{opt}}^{\mathrm{gr}} / c$. Because the nonlinear polarization and the intensity are proportional, this expression can be inserted in Eq. (14), so that in the inhomogeneous term of the equation of motion the decreasing of the intensity is automatically encoded. For a Gaussian pulse the amplitude $A$ can be

\footnotetext{
${ }^{1}$ For the limited number of this contribution we resign to present the corresponding figure.
} 
written as

$$
\begin{gathered}
A(\Omega, z)=\sum_{n=0}^{\infty} a_{n} z^{n} \\
a_{n}= \begin{cases}\mathrm{e}^{-l q z} \sum_{m=1}^{\infty} \frac{p_{n+m}}{b} \frac{(n+m) !}{m !} & \text { if } n \geq 1 ; \\
\mathrm{e}^{-\alpha z / 2} \sum_{m=1}^{\infty} \frac{p_{n+m}}{b} \frac{(n+m) !}{m !} & \text { if } n=0 .\end{cases}
\end{gathered}
$$

with $b=\alpha / 2-\imath \Delta k_{0}$ and $p_{m}=-l \frac{\Omega \chi_{\text {eff }}^{(2)}}{2 k c^{3} n_{0} \varepsilon_{0}} \tilde{I}_{n}$.

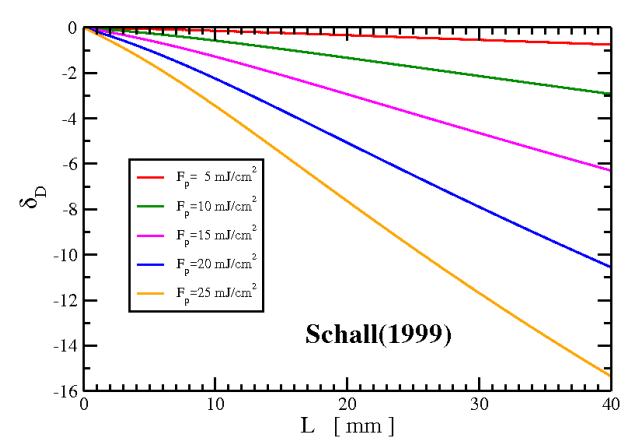

Figure 5: Percentual deviation $\delta_{\mathrm{D}}$ as function of the crystal length $L$ using the parameters set Schall(1999) without FC contribution for different values of the pump fluence as indicated in SVA.

Using this expansion, we can calculate the efficiency $\eta_{\mathrm{D}}$ by including the laser intensity decreasing. We estimate the influence of this effects in SVA using the percentual deviation from the $\eta$ without it $\delta_{\mathrm{D}}=\left(\eta_{\mathrm{D}}-\eta\right) \eta^{-1} \times 100$, that we show in Fig. 5 as function of the crystal length $L$ using the parameters set Schall(1999), without considering the FC contribution, for different values of the pump fluence as indicated.

We note a significant reduction of the efficiency because of the decreasing of the laser pump intensity, in particular for large crystal length and high fluence. In this region, this effect cannot be neglected. Additionally, at fixed $L$, the deviation become stronger by increasing fluence. This suggests that the efficiency as function of the fluence is no linear anymore, but a saturation behavior is expected. The investigations of this dependence are in progress.

\section{CONCLUSION}

The optical properties of the lithium niobate crystal play a crucial role for the generation of $\mathrm{THz}$ pulses and consequently for the planned shot to shot feedback system, which have to perform the synchronization between electron bunch and the ultrashort laser for laser driven plasma wakefield accelerators, with a time resolution of less than $1 \mathrm{fs}$.

We present systematic calculations of the optical properties of the lithium niobate crystal and of their influence on the efficiency of the generation of $\mathrm{THz}$ pulses. We compare different approximation for the modeling of the generation dynamic (SVA vs. second order calculation) as well as for the modeling of the dielectric function. In this way we can clearly show the importance of a consistent description of the optical properties, not only in the $\mathrm{THz}$ range, but also at higher frequencies, the consideration of the complete, i.e. second order, equation of motion for the $\mathrm{THz}$ field as well as the inclusion of the effects of the decreasing intensity of the laser pump.

\section{ACKNOWLEDGEMENT}

The work of S. Mattiello is supported by the German Federal Ministry of Education and Research (BMBF) under contract no. 05K16ROA.

\section{REFERENCES}

[1] A. G. Stepanov, S. Henin, Y. Petit, L. Bonacina, J. Kasparian, and J.-P. Wolf, "Mobile source of high-energy single-cycle terahertz pulses", Appl. Phys. B, vol. 101, no. 1, pp. 11-14, 2010 .

[2] S. Mattiello, H. Schlarb, and A. Penirschke, "Optical rectification for a new shot to shot feedback system for laser-driven plasma wakefield accelerators", in Proc. SPIE 10684, Nonlinear Optics and its Applications (SPIE:18), Strassbourg, France, May 2018, pp. 1068412, doi.org/10.1117/12. 2306429

[3] T. Tajima and J. M. A. Dawson, "Laser Electron Accelerator", Phys. Rev. Lett., vol. 43, no. 4, pp. 267-270, 1979.

[4] K. Ravi, D. Schimpf, and F. Kärtner, "Pulse sequences for efficient multi-cycle terahertz generation in periodically poled lithium niobate", Optics Express, vol. 24, no. 22, pp. 2558225607, 2016.

[5] T. Hattori and K. Takeuchi, "Simulation study on cascaded terahertz pulse generation in electro-optic crystals", Optics Express, vol. 15, no. 13, pp. 8076-8093, 2007.

[6] A. Schneider, M. Neis, M. Stillhart, B. Ruiz, R. Khan, and P. Günter, "Generation of terahertz pulses through optical rectification in organic DAST crystals: theory and experiment", J. Opt. Soc. Am. B, vol. 23, no. 9, pp. 1822-1835, 2006.

[7] K. Vodopyanov, "Optical generation of narrow-band terahertz packets in periodically-inverted electro-optic crystals: conversion efficiency and optimal laser pulse format", Optics Express, vol. 14, no. 6, pp. 2263-2276, 2006.

[8] J. A. Fülöp et al., "Generation of sub-mJ terahertz pulses by optical rectification", Optics Letters, vol. 37, no. 4, pp. 557-559, 2012.

[9] S. Zhong et al., "Optimization of terahertz generation from $\mathrm{LiNbO} 3$ under intense laser excitation with the effect of three-photon absorption", Optics Express, vol. 23, no. 24, pp. 31313-31323, 2015.

[10] L. H. Deng et al., "Improvement to Sellmeier equation for periodically poled LiNbO3 crystal using mid-infrared difference-frequency generation", Optics Communications, vol. 268, no. 1, pp. 110-114, 2006.

[11] M. Schall, H. Helm, and S. R. Keiding, "Far Infrared Properties of Electro-Optic Crystals Measured by THz TimeDomain Spectroscopy", International Journal of Infrared and Millimeter Waves, vol. 20, no. 4, pp. 595-604, 1999. . . 西

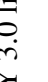

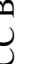

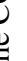

